\title{
Lumbar Spinal Tuberculosis Presenting as Abdominal Pain: Case Report
}

\author{
Zhaoyu Ba ${ }^{1}$ Zhiyao Yong ${ }^{1}$ Weidong Zhao ${ }^{1}$ Bin Shen ${ }^{1}$ Yufeng Huang ${ }^{1}$ Desheng $\mathrm{Wu}^{1}$ \\ ${ }^{1}$ Spine Department, Shanghai East Hospital, Shanghai, China \\ Address for correspondence Dr. Desheng Wu, Spine Department, \\ Shanghai East Hospital, 150, Jimo Road, Shanghai 200120, China \\ Surg J 2015;1:e44-e46. \\ (e-mail: eastspine@yahoo.com).
}

\begin{abstract}
Keywords

- abdominal pain

- lumbar spinal

- tuberculosis

Tuberculosis is a serious disease. Spinal tuberculosis is the most common osseous manifestation of Mycobacterium tuberculosis infection. Early diagnosis through familiarity with its imaging characteristics is essential to permit rapid treatment and prevent potential life-limiting consequences, but its atypical presentations pose diagnostic dilemmas in clinical practice. ${ }^{1,2}$ Although treatable, it continues to cause significant mortality and morbidity. In the current literature, a patient with spinal tuberculosis and presenting only with abdominal pain is very rare. We report a 50 -year-old woman with abdominal pain as a presenting symptom of lumbar spinal tuberculosis.
\end{abstract}

More than 230 years after the characteristic clinical features of tuberculosis spondylitis were first described, this serious infection can still present diagnostic dilemmas in clinical practice. Atypical presentations of spinal tuberculosis mimicking malignancy have been described. We report a 50-year-old woman with abdominal pain as a presenting symptom of lumbar spinal tuberculosis. She presented with chronic intermittent abdominal pain of 6 months' duration, without low-grade fever and weight loss. There were no bowel/bladder complaints, vomiting, or worm infestation. Magnetic resonance imaging of the lumbar spine showed the $L 3, L 4$, and $L 5$ vertebrae bodies had been destroyed and disease had invaded the lumbar spinal canal. There was a giant abscess in the right musculus psoas major. The C-reactive protein level was $130 \mathrm{~g} / \mathrm{L}$, and the erythrocyte sedimentation rate was $165 \mathrm{~mm} / \mathrm{h}$. A diagnosis of lumbar spinal tuberculosis with abdominal pain was made. After 3 weeks of antituberculosis treatment, an operation was performed to debride the necrotic tissues and reconstruct the L3 vertebrae through an anterior approach combined with a posterior approach to establish the spine stability. On 6-month follow-up, the patient had recovered from the operation and had no focal neurologic deficit.

\section{Case Report}

The 50-year-old woman presented with chronic intermittent abdominal pain of more than 6 months' duration, but with no low-grade fever or weight loss. She reported that she used to hold her lower abdomen or sleep for a while during episodes of pain, and the pain would fade. There were no bowel or bladder complaints, vomiting, or worm infestation. Complete blood counts, serum electrolytes, serum calcium, serum amylase, ultrasonography of the abdomen, liver and renal function tests, and stool and urine examinations were normal. The C-reactive protein level was $130 \mathrm{~g} / \mathrm{L}$, and the erythrocyte sedimentation rate was $165 \mathrm{~mm} / \mathrm{h}$. On systemic examination, percussion test of the lumbar spine was positive. Neurologic examination revealed hyper-reflexia of both patella tendons. All four limbs had normal strength. Deep tendon reflexes in both knee and ankle joints were brisk. The rest of the examination was normal.

The magnetic resonance imaging of the lumbar spine showed an occupying lesion at the vertebrae bodies of L2, L3, and L4 and a giant abscess in the right musculus psoas major (-Fig. 1). The computed tomography scans revealed that the giant abscess extended to the lesser trochanter received

June 23, 2015

accepted

July 22, 2015

published online

August 21, 2015
DOI http://dx.doi.org/

$10.1055 / \mathrm{s}-0035-1563612$

ISSN 2378-5128.
Copyright $\odot 2015$ by Thieme Medical

Publishers, Inc., 333 Seventh Avenue,

New York, NY 10001, USA.

Tel: +1(212) 584-4662.
License terms

$(\circledast) \Theta \circledast$ 


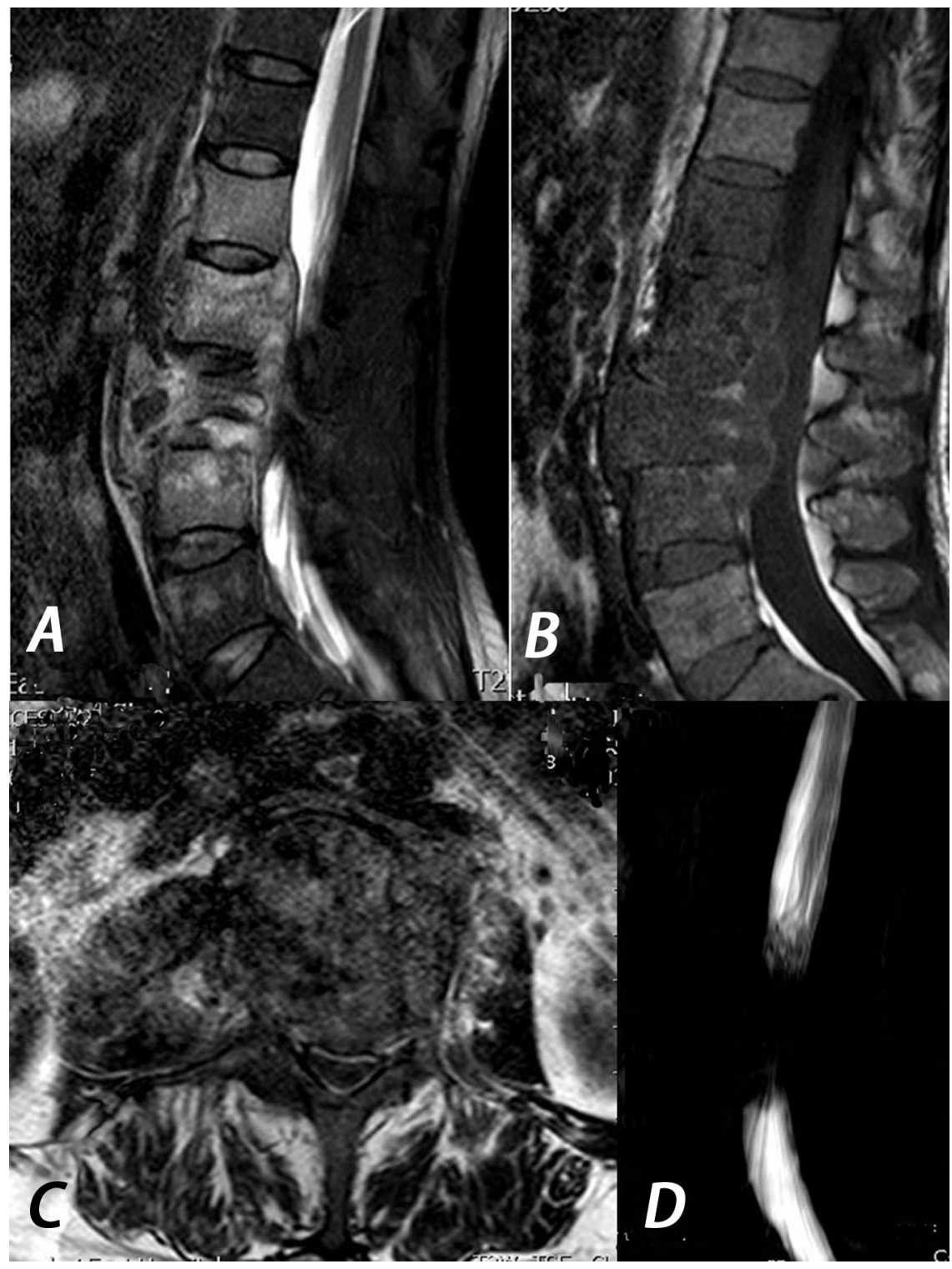

Fig. 1 (A, B) Magnetic resonance T2- and T1-weighted images in sagittal view showed involvement of L2-L4 vertebrae with reduced disk space and the lumbar spinal canal. (C, D) Axial section of the same patient showed destruction of L2-L4 vertebrae with a giant cold abscess on the right side and magnetic resonance myelogram showed the lumbar spinal canal was compression remarkable.

(-Fig. 2). A diagnosis of spinal tuberculosis with abdominal pain was made. Conservative treatment was planned. Antituberculosis treatment with isoniazid, rifampicin, ethambutol, and pyrazinamide was initiated. After 3 weeks of antituberculosis treatment, the patient received an operation. We removed all the granulation tissue, pus, and necrotic bone and reconstructed the L3 vertebrae to restore stability to the lumbar spine (-Fig. 3). The C-reactive protein rate was $110 \mathrm{~g} / \mathrm{L}$, and the erythrocyte sedimentation rate was $50 \mathrm{~mm} / \mathrm{h}$ 3 days postoperatively. At the last follow-up 6 months after the operation, she was still asymptomatic and without any neurologic deficit.

\section{Discussion}

Tuberculosis of the spine can cause serious morbidity, such as permanent neurologic deficits and severe deformity.

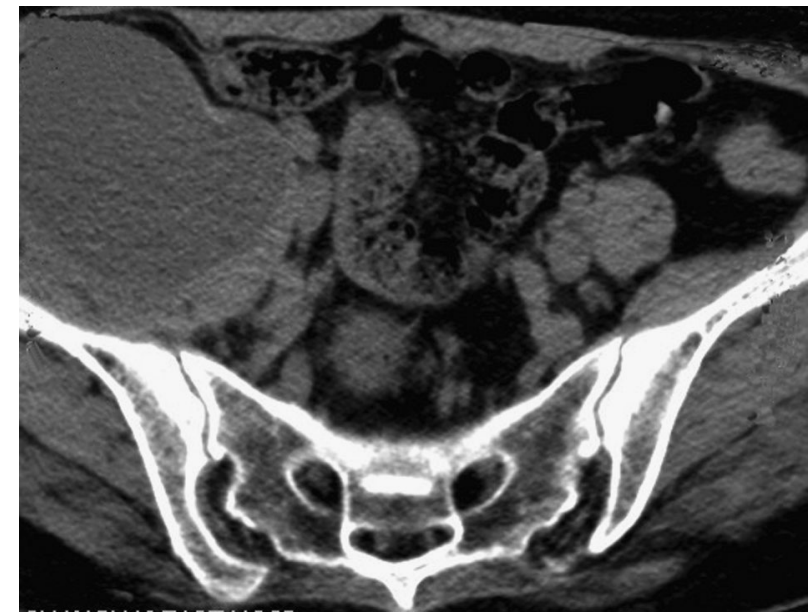

Fig. 2 Computed tomography revealed a giant cold abscess on the right lliac fossa. 


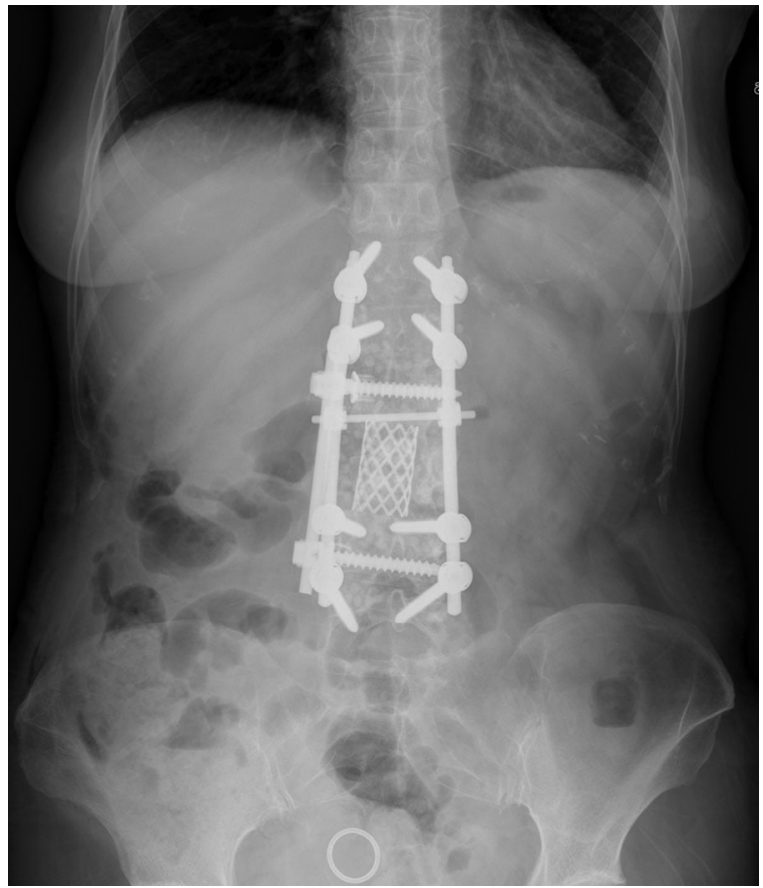

Fig. 3 We used an anterior approach combined with the posterior to reconstruct the $\mathrm{L} 3$ vertebrae and restored the lumbar spine stability.

Accurate diagnosis as early as possible is essential. Any part of the spine can be involved, but there is a predilection for lower thoracic and upper lumbar spine vertebrae. ${ }^{3}$ Only $5 \%$ of spinal tuberculosis cases involve the paraspinal space. Tuberculosis of the spine accounts for 50 to $60 \%$ of all skeletal tuberculosis, and $75 \%$ of cases occur in the thoracolumbar spine. Nearly $50 \%$ of cases have three levels affected. ${ }^{4,5}$

The onset of symptoms is always insidious, and the disease progresses slowly. Low-grade fever, weight loss, pain, rigidity, deformity, cold abscess, and paraplegia are the classic symptoms as described by Iqbal et al. ${ }^{6}$ The typical paradiscal tubercular lesion is well described and easily recognized and treated, ${ }^{7}$ but our patient had abdominal pain as a rare presenting symptom of spinal tuberculosis. Such rare symptoms may require extensive testing to rule out other disease processes, which may cause late or missed diagnosis accompanied by irreversible neurologic sequelae including paraplegia or loss function of the sphincter muscles. In this patient, lumbar spinal tuberculosis caused abdominal pain, leading to a delay in the diagnosis. Care must be taken to obtain magnetic resonance imaging for patients with abdominal pain and no other symptoms.

To the best of our knowledge, there are only three theories to explain the phenomenon of spinal referred pain: the axon reflex theory, the convergence theory, and the hyperexcitability theory. The axon reflex theory considers that certain primary sensory neurons have axons innervating both somatic and visceral targets, leading to confusion as to the source of afferent activity. ${ }^{8}$ The convergence theory agrees that the afferent nerves from one region converge in the spinal cord with afferent nerves from another region onto a common second-order neuron, allowing misinterpretation of the source of pain by the central nervous system. ${ }^{9}$ The hyperexcitability theory suggests that the referred pain occurs via cross-connections between second-order neurons supplying the different regions, but only when the input reaches a certain threshold. ${ }^{10}$ Feinstein et al found common patterns of referred pain following irritation of thoracic and lumbar spinal somatic structures. ${ }^{11}$ The referred pain is always felt as deep, dull, or aching and is diffuse in its distribution. Our case had referred pain possibly due to involvement of the $\mathrm{L} 2$ vertebrae.

We want to highlight this atypical presentation of spinal tuberculosis. Spinal tuberculosis should be considered in patients presenting with abdominal pain.

\section{Conflict of Interest}

This work was funded by Key Discipline Construction Project of Pudong Health Bureau of Shanghai.

\section{Acknowledgments}

The authors thank the patient for permission to report the case and doctors Zhen Zhang, Yan Zhang, Ju Liu, and Yang Meng for their help.

\section{References}

1 Ringshausen FC, Tannapfel A, Nicolas V, et al. A fatal case of spinal tuberculosis mistaken for metastatic lung cancer: recalling ancient Pott's disease. Ann Clin Microbiol Antimicrob 2009;8:32

2 Chakraborty PP. Deviated tongue: the presenting manifestation of spinal tuberculosis. Indian J Pediatr 2009;76(9):967-969

3 Tuli SM. Tuberculosis of the spine. In: Tuberculosis of the Skeletal System. 3rd ed. Noida, India: Jaypee Brothers Medical Publishers; 2004:191-198

4 al Arabi KM, al Sebai MW, al Chakaki M. Evaluation of radiological investigations in spinal tuberculosis. Int Orthop 1992;16(2):165-167

5 Smith AS, Weinstein MA, Mizushima A, et al. MR imaging characteristics of tuberculous spondylitis vs vertebral osteomyelitis. AJR Am J Roentgenol 1989;153(2):399-405

6 Iqbal M, Bilal S, Sarwar S, Murphy R. Tuberculosis abscess causing spinal cord compression: a rare presentation. Ir J Med Sci 2011; 180(2):625-626

7 Pande KC, Babhulkar SS. Atypical spinal tuberculosis. Clin Orthop Relat Res 2002;(398):67-74

8 McMahon SB. Mechanism of cutaneous, deep, and visceral pain. In: Wall PD, Melzack R, eds. Textbook of Pain. 3rd ed. Edinburgh, UK: Churchill Livingstone; 1994:129-151

9 Harding G, Yelland M. Back, chest and abdominal pain-is it spinal referred pain? Aust Fam Physician 2007;36(6):422-423, 425, 427429

10 Arendt-Nielsen L, Svensson P. Referred muscle pain: basic and clinical findings. Clin J Pain 2001;17(1):11-19

11 Feinstein B, Langton JNK, Jameson RM, Schiller F. Experiments on pain referred from deep somatic tissues. J Bone Joint Surg Am 1954;36-A(5):981-997 